\title{
Permeability Studies on the Ocular Absorbance of Nanostructured Materials Across the Cornea
}

\author{
S. BLASS ${ }^{1}$, B. TeUbl ${ }^{1}$, E. FRÖHLICH ${ }^{2}$, C. MEINDL ${ }^{2}$, D. F. RABENSTEINER ${ }^{3}$, \\ G. Trummer ${ }^{3}$, O. SCHMUt ${ }^{3}$, A. Zimmer ${ }^{1}$, E. ROBLegG ${ }^{1}$ \\ ${ }^{1}$ Institute of Pharmaceutical Sciences/ Pharmaceutical Technology, Karl-Franzens University Graz, Austria \\ ${ }^{2}$ Center of Medical Research, Medical University Graz, Austria \\ ${ }^{3}$ Department of Ophthalmology, Medical University Graz, Austria \\ E-mail: sandra.blass@uni-graz.at (S. Blass)
}

Sci Pharm. 2010; 78: 678

doi:10.3797/scipharm.cespt.8.PNM08

Introduction: The eye provides a variety of defense mechanisms, which protect the organ against numerous different substances. These mechanisms include the bony orbit, the eyelids and cilia, the tear film and nasolacrimal drainage, corneal and conjunctival epithelium, and a submucosal secretory immune defense. In order to overcome the strong physiological barriers, new drug delivery systems have been established, including the development of nanocarriers. However, it is known that nanostructured materials are able to invade cellular structures and trigger toxic reactions. Methods: For the establishment of an in vitro system, fluorescence-labelled carboxyl polystyrene particles (CPP $20 \mathrm{~nm}$ and $200 \mathrm{~nm}$ ) were characterized in terms of their physico-chemical properties (size, distribution, agglomeration, surface charge) in different biological media (i. e. MQ-water, $0.9 \% \mathrm{NaCl}$ and tear fluid) using a ZetaSizer Nano-ZS (Malvern). Ex vivo permeability studies were performed with static Franz diffusion cells. Donor and receiver compartment were separated by insertion of three weeks cultured excised human corneae. CPP $(20 \mathrm{~nm})$ were suspended in physiological $\mathrm{NaCl}$, applied in concentrations of $100 \mu \mathrm{g} / \mathrm{ml}$ and $250 \mu \mathrm{g} / \mathrm{ml}$ and incubated for 6 hours. Samples were withdrawn from the sampling port of each cell and fluorimetrically analyzed. After 6 hours the tissue was fixed, frozen, sliced into $10 \mu \mathrm{m}$ thin slides and observed with a fluorescence microscope. Results: Size and surface charge of the particles differed according to the medium. Particles suspended in tear fluid showed reduced surface charges and diameters three times higher, compared to those, suspended in $\mathrm{MQ}$-water and $0.9 \% \mathrm{NaCl}$. This increase in particle size can not only be explained by partial aggregation of the particles but also by the fact that in biological fluids, proteins attach to the nanoparticle surface, which leads to the so called 'protein corona'. Histopathological Analysis showed that corneal epithelium and endothelium detached during prolonged storage and incubation. Fluorescent particles were seen in the entire corneal stroma. Conclusion: $20 \mathrm{~nm}$ CPP can penetrate through Bowman's and Descement membrane and the entire corneal stroma. Corneae with shorter culturing times will be used for the assessment of the epithelial barrier function.

[1] Oberdörster G, Oberdörster E, Oberdörster J. Nanotoxicology: An emerging discipline evolving from studies of ultrafine particles. Environ Health Perspect. 2005; 113: 823-39. doi:10.1289/ehp.7339 